\title{
Investigations of the electron temperature profiles at the WEGA stellarator
}

\author{
Kinga Horvath $\S$, Johann Lingertat, Matthias Otte and \\ Friedrich Wagner \\ Max-Planck-Institut für Plasmaphysik, Euratom Association, TI Greifswald, \\ Wendelsteinstraße 1, 17491 Greifswald, Germany
}

\begin{abstract}
This paper presents a study of the hollow bulk electron temperature profiles measured in the low-temperature $(2-15 \mathrm{eV})$ plasmas of the WEGA stellarator. For this the global power balance equation was solved for the bulk electrons. The observed hollow temperature profiles could be reproduced satisfactorily assuming that the bulk electrons are heated through energy transfer from fast electrons and that the parallel heat conduction in the scrape-off layer is determined by the potential drop in the sheath in the vicinity of the wall. These results imply that the cause of the hollow shape of bulk electron temperature profiles is the heating of them through collisions with fast electrons produced by the heating method.
\end{abstract}

PACS numbers: 52.25.Xz, 52.55.Dy, 52.55.Hc

$\S$ To whom correspondence should be addressed (horvathkinga@yahoo.com) 


\section{Introduction}

The WEGA device is a classical stellarator operated in the Max-Planck-Institut für Plasmaphysik (IPP), Greifswald (Germany). Its main goals are: training of students, diagnostics development and testing of the infrastructure for the advanced optimised stellarator W7-X [1] and basic research in plasma physics.

A typical property of the electron cyclotron resonance (ECR) heated WEGA plasmas is the hollow shape of the bulk electron temperature profiles. Since such a shape of the electron temperature profile is not efficient for confinement, it is important to find the reason for its formation. In the present paper these hollow bulk electron temperature profiles are studied solving the global power balance equation of electrons.

The article is organized as follows. In Section 2, an overview of the WEGA stellarator is given and some relevant measurement results are presented. Section 3 is devoted to the study of hollow electron temperature profiles. The last section contains a discussion of the results and conclusions.

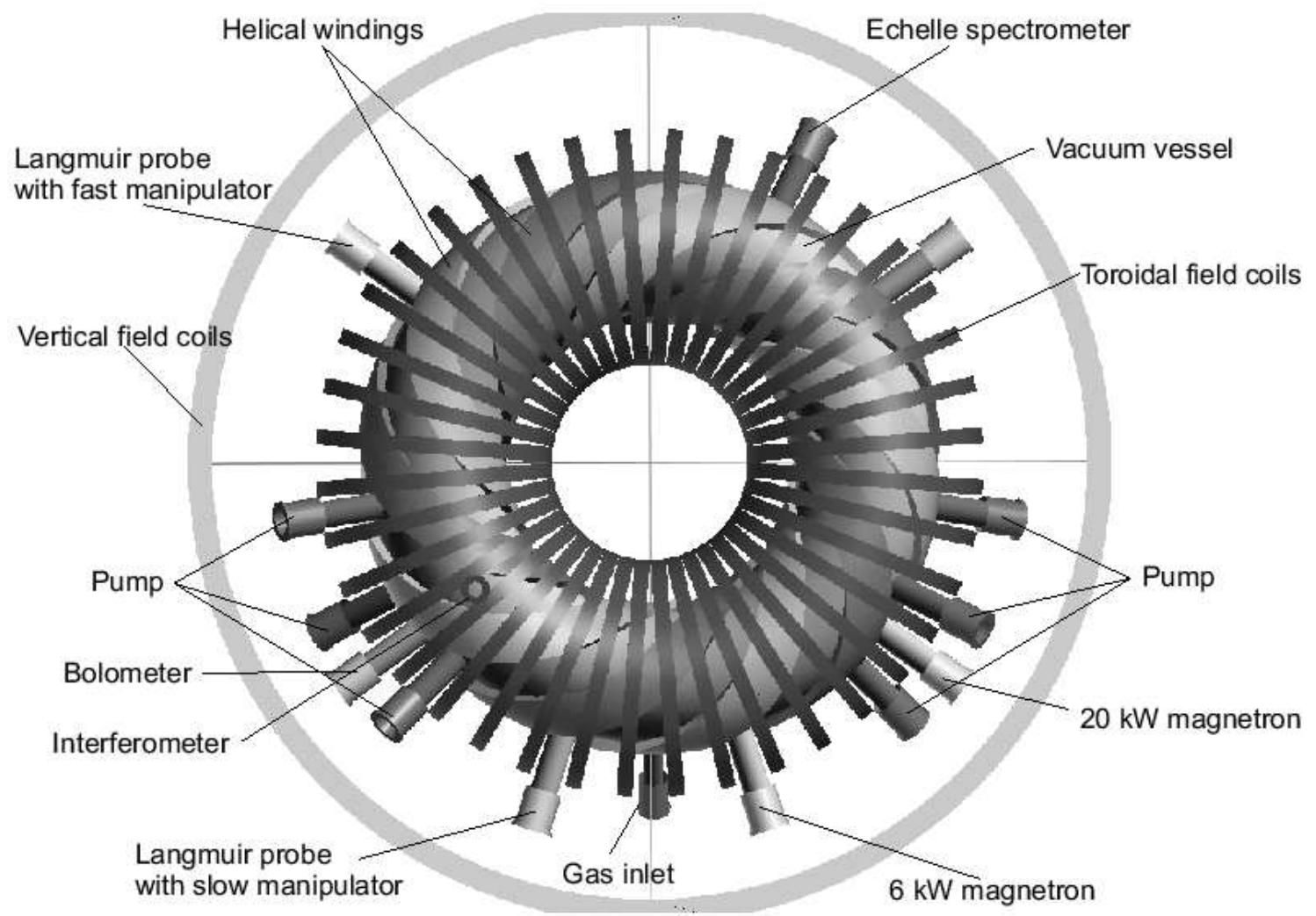

Figure 1. Top view of the WEGA stellarator. The location of the pumps, gas inlet, heating and diagnostics, as well as the magnetic coils are indicated. 


\section{The WEGA Stellarator}

The WEGA stellarator was originally built in Grenoble (France) in the 1970s. In the 1980s it was installed at the university of Stuttgart (Germany) and it was moved to the Greifswald branch of IPP in 2000, where it was modernised.

The 40 planar coils and the 2 pairs of helical windings of WEGA produce magnetic field lines with $l=2$ poloidal and $m=5$ toroidal periodicity. The vessel dimensions are: $R_{\text {vessel }}=0.72 \mathrm{~m}, r_{\text {vessel }}=0.19 \mathrm{~m}$. A technical sketch of WEGA is shown in figure 1 .

The plasma is ignited and heated through electron cyclotron resonance heating (ECRH). The applied O-mode wave has a frequency of $2.45 \mathrm{GHz}$. Two magnetrons are available with a maximum heating power of $6 \mathrm{~kW}$ and $20 \mathrm{~kW}$, respectively, and can be operated independently. The coupling of the wave to the plasma is achieved using an optimised circular two-slot antenna [2].

The diagnostics used in the present work are: Langmuir and emissive probes, singlechannel interferometer, emission spectroscopy and a baratron. The further analysis relies on parameter profiles measured with the help of the scanning Langmuir probe. The other diagnostics were mostly used for cross-check purposes and the interferometer for the calibration of the electron density measured by the electrical probe.

The Langmuir probe characteristics observed at WEGA have a common anomalous feature: the ion current does not saturate. Many reasons of this phenomenon were eliminated and a possible explanation was found: the presence of non-thermal electrons [3]. Using a two-temperature Maxwellian distribution function of electron energy, the measured probe characteristics could be fitted and the following parameters were determined: $T_{e s}\left(T_{e f}\right)$ - temperature of the slow (fast) electron component, $f_{T}=T_{e f} / T_{e s}, f_{n}=n_{e f} / n_{e s}\left(n_{e f}, n_{e s}-\right.$ densities of the fast and slow electron components), $n_{e}$ - total electron density, $V_{p}$ - plasma potential, $V_{f}$ - floating potential.

Figure 2 shows typical profiles of plasma parameters. The main properties of these profiles are: $n_{e}$ profiles are usually flat or peaked, $T_{e s}$ profiles generally hollow. The $T_{e f}$ profiles have generally two peaks: one in the plasma centre and one in the scrape-off layer (SOL). It is also an interesting feature, that the $V_{f}$ profiles always show a minimum close to the last closed flux surface (LCFS) and $f_{n}$ usually has a peak at this position. It should be mentioned that the parameter profiles were measured in a steady state phase of the plasma development. This was observed from the interferometer signal.

For the analysis of the bulk electron temperature profiles, the knowledge of the vacuum flux surfaces is required. The flux surfaces were measured using the fluorescence technique [4] and agreed well with calculated ones using a magnetic field line tracing code [5]. The existence of magnetic confinement of WEGA plasmas at low temperature and high collisionality was also demonstrated. A first evidence was the above mentioned phenomenon: minima of $V_{f}$ profiles near the LCFS in all discharges. This can be clearly seen in figure 3. Another evidence is related to the measurements of plasma parameters for different magnetic configurations. From flux surface measurements and calculations it is known that the plasma can be shifted by applying a vertical magnetic field [6], 

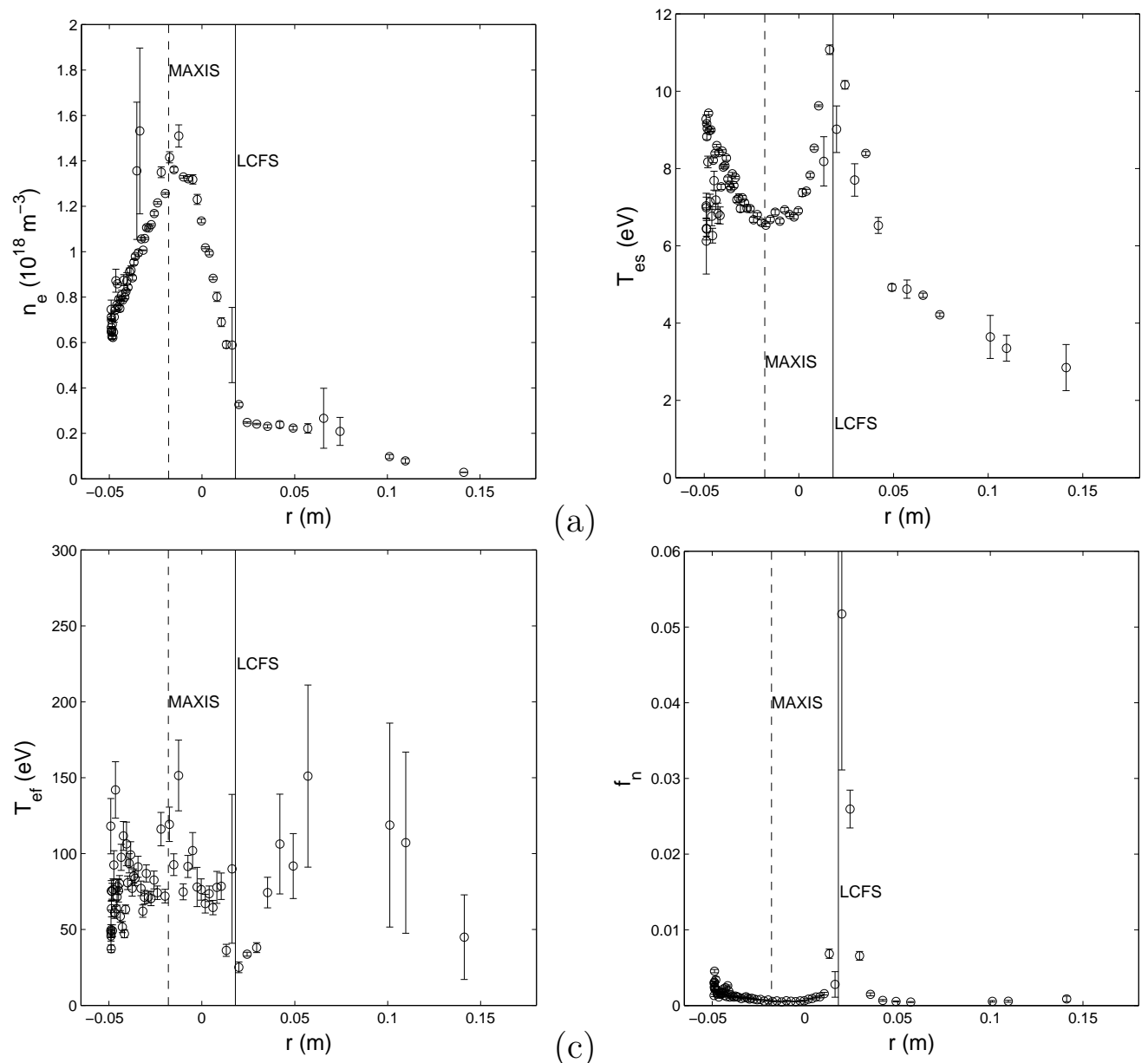

(a)

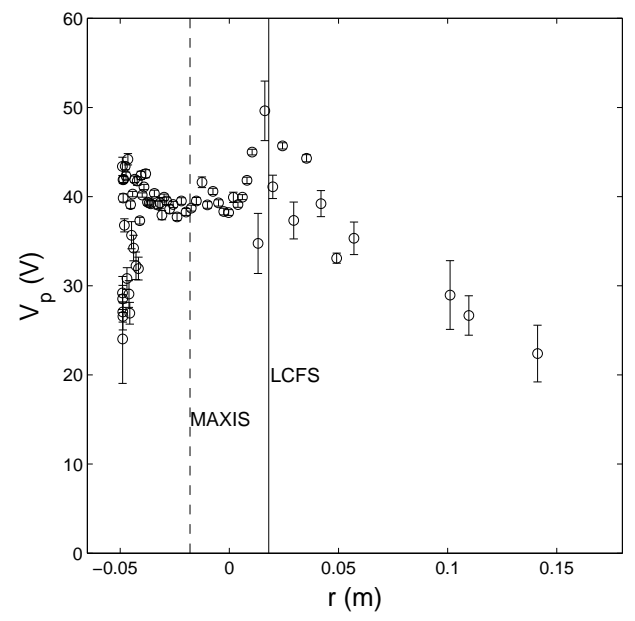

(c)

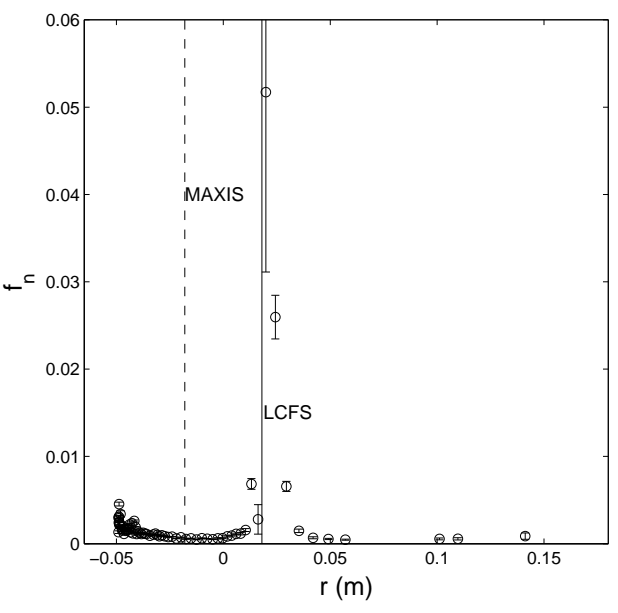

(d)

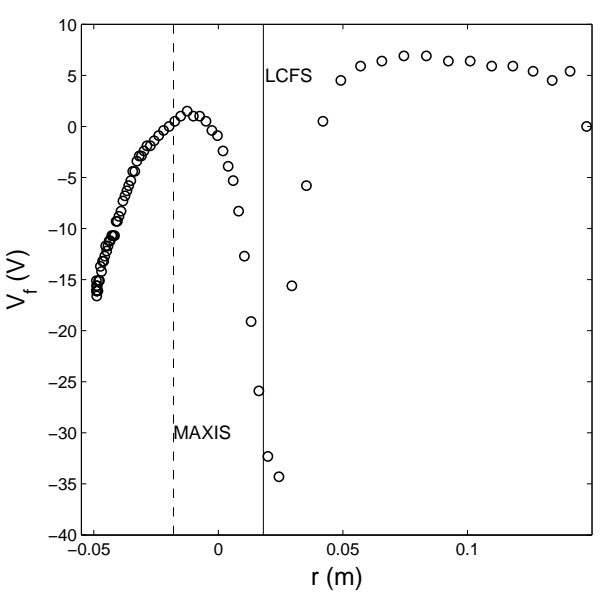

Figure 2. Typical radial profiles measured in WEGA plasmas: (a) $n_{e}-$ electron density; (b) $T_{e s}$ - temperature of slow electrons; (c) $T_{e f}$ - temperature of fast electrons; (d) $f_{n}=n_{e f} / n_{e s}$ - fraction of density of fast electrons; (e) $V_{p}$ - plasma potential; (f) $V_{f}$ - floating potential. The dashed and solid vertical lines show the positions of the magnetic axis and last closed flux surface, respectively. (Ar, toroidal magnetic field at the centre of the vessel $B_{0}=52.5 \mathrm{mT}$, heating power $P_{m w} \approx 6 \mathrm{~kW}$, neutral pressure $p_{n}=1.6 \times 10^{-3} \mathrm{~Pa}$ ) 


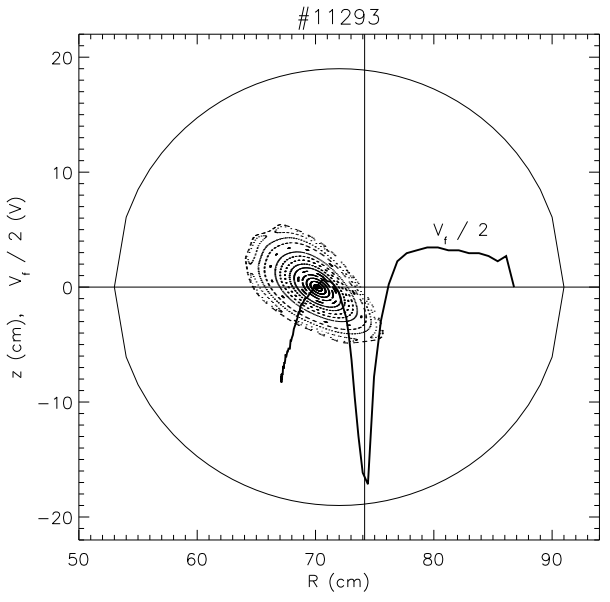

Figure 3. Example of $V_{f} / 2(R)$ profile for $z=0$ and the poloidal cross-section of the flux surfaces. $R$ is the major radial and $z$ is the vertical coordinate.

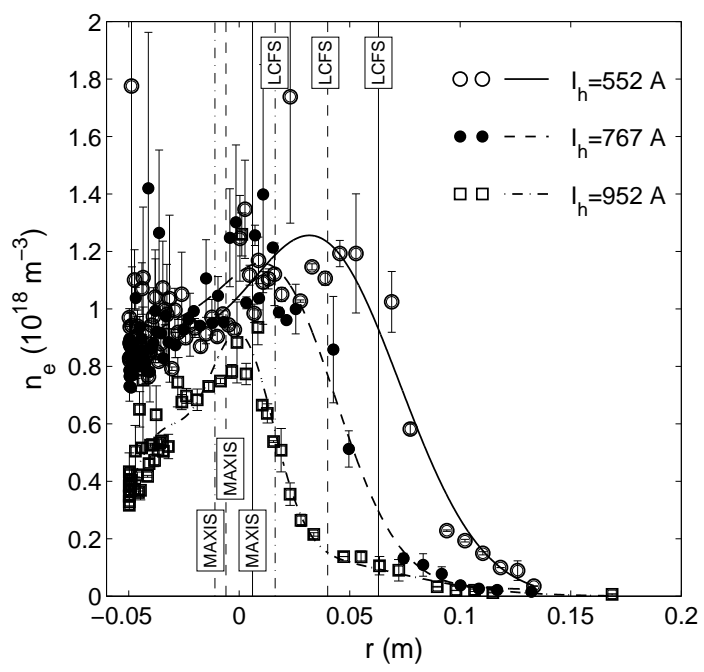

Figure 4. Electron density profiles measured in plasmas using different magnetic configurations.

by changing the helical field (at constant vertical magnetic field). The latter behaviour could be observed in the shape of the electron density and pressure profiles, too. For example, $n_{e}$ profiles measured for plasmas with different helical field current $\left(I_{h}\right)$ are presented in figure 4. The vertical lines indicate the positions of the magnetic axis and the LCFS calculated using the field line tracing code. The shift of the plasma toward the edge with decreasing $I_{h}$ is indicated both by the measured profiles and the calculations. The largest density gradients are near the LCFS. As known from flux surface calculations, with increasing $I_{h}$ the plasma volume is decreasing. This can be observed from the calculated positions of the LCFS, as well as from the $n_{e}$ profiles.

\section{Simulation of hollow $T_{e s}$ profiles}

The $T_{e s}$ profiles were calculated by solving the global power balance equation for the slow electrons inside and outside the LCFS,

$$
P_{a b s}=P_{\text {transp }}+P_{\text {loss }},
$$

where $P_{a b s}$ is the absorbed power, $P_{t r a n s p}$ is the power transported by conduction and convection and $P_{\text {loss }}$ represents power sinks through atomic processes.

\subsection{Power balance inside the LCFS}

If the power balance is valid inside each flux surface, it can also be written in terms of power densities $\left(Q^{W}\right)$ as

$$
Q_{\text {abs }}^{W}\left(r_{e f f}\right)=Q_{\text {transp }}^{W}\left(r_{e f f}\right)+Q_{\text {loss }}^{W}\left(r_{e f f}\right) .
$$


$r_{\text {eff }}$ is the effective radial coordinate, which was calculated as the radius of a circle with the same area as the flux surface at the position of interest.

In the following, the power densities from Eq. (2) will be discussed.

A main assumption of the present calculations was that $Q_{a b s}^{W}=Q_{f s}^{W}$, which means that the absorbed power density is the power density transfered from hot to cold electrons through collisions. This can be written as

$$
Q_{f s}^{W}=\frac{\frac{3}{2} n_{e f}\left(T_{e f}-T_{e s}\right)}{\tau_{e q}^{e f e s}},
$$

where $\tau_{e q}^{e f e s}$ is the fast electron - slow electron energy relaxation time,

$$
\tau_{e q}^{e f e s} \approx\left(\frac{4 \pi \epsilon_{0}}{e^{2}}\right)^{2} \frac{3 m_{e} T_{e f}^{3 / 2}}{8 \sqrt{2 \pi m_{e}} n_{e s} \ln \Lambda} .
$$

( $\ln \Lambda$ is the Coulomb logarithm.)

The transported power density is the sum of a convective,

$$
Q_{c o n v, \perp}^{W}=-\nabla\left[\chi_{\perp} n_{e s} \nabla\left(k_{B} T_{e s}\right)\right]
$$

and a conductive term,

$$
Q_{\text {cond, } \perp}^{W}=\nabla\left(\frac{3}{2} k_{B} T_{e s} \Gamma\right)
$$

The power densities for the parallel transport are negligible inside the LCFS, since the plasma parameters are assumed to be constant on flux surfaces. $\chi_{\perp}$ is the perpendicular thermal diffusion coefficient, which was assumed to be constant. $\Gamma=-D \nabla n_{e s}$ is the particle flux. The particle diffusion coefficient $D$ was determined by solving the particle balance equation.

$Q_{\text {loss }}^{W}$ can be the power density of ionisation $\left(Q_{i o n}^{W}\right)$, of excitation of neutrals $\left(Q_{\text {exc0 }}^{W}\right)$, of excitation of ions $\left(Q_{\text {exci }}^{W}\right)$, of recombination $\left(Q_{r e c}^{W}\right)$ and of energy transfer from electrons to ions $\left(Q_{e s i}^{W}\right)$. The latter two power densities are always small for the WEGA plasmas.

\subsection{Power balance outside the LCFS}

Equation (2) is valid also outside the LCFS. However, $r_{\text {eff }}$ was determined in a different way from that used for the confined region, because closed flux surfaces do not exist in the SOL. It was assumed that in the SOL the plasma parameters decrease radially independently on the poloidal angle. Thus, surfaces with constant parameters were determined. $r_{\text {eff }}$ was calculated as the radius of a circle with the same area as these surfaces.

It was supposed $Q_{a b s}^{W}=Q_{f s}^{W}$ also in this region and $Q_{\text {loss }}^{W}$ was determined as described above. However, the transported power density had to be calculated in a different way. The convective transport is usually small in the SOL, therefore it was neglected. The parallel conductive power density $\left(Q_{\text {cond,\|, }}^{W}\right)$ was taken into account, since this term is usually the dominant one in the power balance outside the LCFS.

Two approaches were applied to determine $Q_{c o n d, \|}^{W}$, which represent two extreme cases. 
The first assumption was that $T_{\text {es }}$ decreases toward the wall reaching the value 0 and the parallel conduction is determined by this decay. Then

$$
Q_{\text {cond, }, \|}^{W}=\frac{q_{\text {cond, }, \|}}{L} n_{x} \text {. }
$$

$n_{x}$ is the number of $\mathrm{X}$ points, and it is equal to 2 at the poloidal periodicity $l=2$. $L$ is the connection length of the magnetic field lines to the wall and it is defined as $L=\pi R_{\text {vessel }} \frac{2 \pi}{\iota} . \iota$ is the rotational transform. $q_{\text {cond, } \|}$ is the heat flux at the position for which the power balance is written,

$$
q_{\text {cond }, \|}=-\chi_{\|} n_{e s} \frac{\mathrm{d}\left(k_{B} T_{e s}\right)}{\mathrm{d} x},
$$

where $\chi_{\|}$is the parallel thermal diffusion coefficient which is a function of $T_{e s}$,

$$
\chi_{\|}=\chi_{0} T_{e s}^{5 / 2} .
$$

Finally, the expression for the parallel conductive power density for this first approach is

$$
Q_{\text {cond, }, \|}^{W}=\frac{2}{7} \chi_{0} n_{e s} \frac{1}{L^{2}} k_{B} T_{e s}^{7 / 2} n_{x}
$$

The second ansatz to obtain the parallel heat conduction in the SOL was to assume that $T_{e s}$ does not decrease along the magnetic field lines, and $Q_{\text {cond, } \|}^{W}$ is determined by the potential drop in the sheath near the wall. In this case the parallel heat flux varies from zero to the value of the heat flux at the sheath edge $q_{s e}$,

$$
Q_{\text {cond, }, \|}^{W}=\frac{q_{s e}}{L}
$$

with

$$
q_{s e}=\delta_{e} k_{B} T_{e s} \Gamma_{s e} .
$$

$\delta_{e}$ is a factor which characterises the potential drop in the sheath and presheath. It has different values for the various gas types: for $\mathrm{Ar}$ it is $\approx 11$, for $\mathrm{He}$ is $\approx 5.5$ and for $\mathrm{H}$ is $\approx 5[7] . \Gamma_{s e}$ is the particle flux at the sheath edge and can be calculated as

$$
\Gamma_{s e}=n_{e s} c_{s}
$$

where the ion acoustic velocity is $c_{s}=\sqrt{k_{B} T_{e s} / m_{i}}$. The final form of $Q_{\text {cond, } \|}^{W}$ for this regime is

$$
Q_{\text {cond, } \|}^{W}=\frac{\delta_{e} k_{B} T_{e s} \Gamma_{s e}}{L} .
$$

It should be mentioned that also $\chi_{\perp}$ was an unknown parameter. For simplicity it was assumed constant, since these calculations had a qualitative aspect only.

Equation (2) was solved with respect to $T_{e s}$ and $\chi_{\perp}$ using a $4^{\text {th }}$ order Runga-Kutta differential equation solving method. 


\subsection{Boundary conditions}

Because of the profile symmetry, $\mathrm{d} T_{e s} / \mathrm{d} r_{\text {eff }}\left(r_{\text {eff }}=0\right)=0$. The initial value for the computation, $T_{e s}\left(r_{\text {eff }}=0\right)$ was taken from measurements.

Two boundary conditions were used at the position of the LCFS to connect the results obtained from solving the balance equation inside and outside the LCFS. $T_{e s}$ values at this position obtained as a solution from the two regions had to be equal. The same was valid for the perpendicular heat fluxes,

$$
q_{c o n d, \perp L C F S}+q_{c o n v, \perp} \perp_{C F S}=\left(-n_{e s} \chi_{\perp} \frac{\mathrm{d} k_{B} T_{e s}}{\mathrm{~d} r_{e f f}}\right)_{L C F S},
$$

where the left-hand side represents the perpendicular heat flux coming from the confined region $\left(q_{\text {cond }, \perp}{ }_{L C F S}, q_{\text {conv }, \perp}{ }_{L C F S}\right.$ are the perpendicular conductive and convective heat fluxes at the LCFS determined by solving Eq. (2) inside the LCFS) and the right-hand side is the perpendicular heat flux which is going into the SOL.

Usually, $\mathrm{d} T_{e s} / \mathrm{d} r_{\text {eff }}$ near the wall can be assumed to be zero. However, in our case no measurements of the plasma parameters could be done in the vicinity of the wall. Therefore, the simulation was done in the region with available data and the measured $\mathrm{d} T_{e s} / \mathrm{d} r_{\text {eff }}$ at the last point in front of the wall was used as a boundary condition. At this position - still relative close to the wall, the derivative of $T_{e s}$ had a quite small value.

\section{Results and discussion}

The results of the simulations are presented in figures 5 and 6 . The first graph shows that the $T_{e s}$ profile, calculated using the first approach (the parallel heat conduction in the SOL is determined by the temperature decrease along the magnetic field lines), is in disagreement with the measured one. For the second approach — parallel heat conduction determined by the potential drop in the sheath - the simulated hollow $T_{e s}$ profile agrees well with the observed one. Also the $\chi_{\perp}$ values determined using the first model was unrealistically high, but using the second one it was in the expected range $\left(\approx 10 \mathrm{~m}^{2} / \mathrm{s}\right)$.

The above presented results give an explanation for the existence of hollow $T_{\text {es }}$ profiles in WEGA plasmas. The agreement between the simulated and measured $T_{\text {es }}$ profiles implies that the underlying assumptions describe well the existing plasma conditions. Thus, it can be concluded that in the case of hollow $T_{e s}$ profiles the slow electrons are mainly heated by energy transfer from fast electrons. The shape of the power density profile transfered from hot to slow electrons is hollow, which explains the formation of bulk electron temperature peaks at the edge of the confined plasma region.

Regarding the other main assumption of the simulations - parallel heat conduction in the SOL determined by the sheaths potential drop - this was also adequate for the studied hollow profiles. 


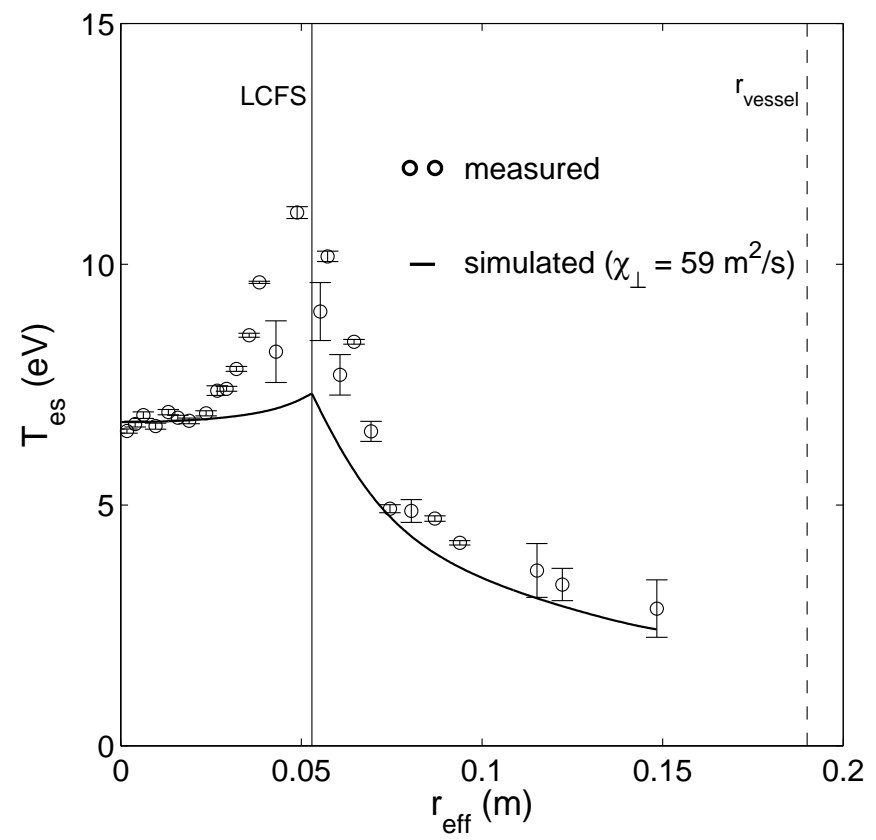

Figure 5. Comparison of the measured $T_{e s}$ profile (circles) with that resulted as a solution (line) of the power balance equations inside and outside LCFS with the assumption that outside the LCFS $T_{e s}$ decreases along the magnetic field lines reaching zero at the wall.

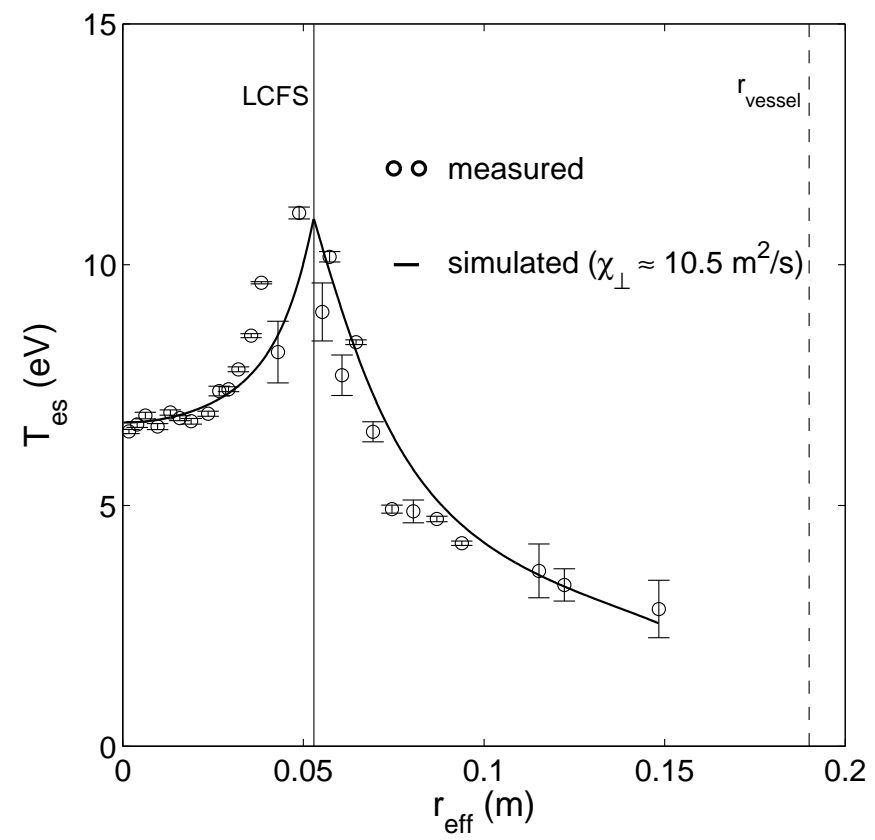

Figure 6. Comparison of the measured $T_{e s}$ profile (circles) to that resulted as a solution (line) of the power balance equations inside and outside LCFS with the assumption that $T_{e s}$ is constant along the magnetic field lines and the parallel heat flux is controlled by the potential drop in the sheath. 


\section{Acknowledgments}

The authors wish to thank H. Laqua and R. Schneider for very helpful discussions and are grateful to D. Aßmus and N. Paschkowski for technical support.

\section{References}

[1] Lotz W, Nührenberg J and Schwab C 1990 Plasma Physics and Controlled Nuclear Fusion Research (International Atomic Energy Agency, Vienna, 1991) p 603

[2] Podoba Y, Horvath K, Laqua H P, Lingertat J, Otte M and Wagner F $200410^{\text {th }}$ International Conference and School on Plasma Physics and Controlled Fusion (Alushta, Ukraine)

[3] Horvath K, Lingertat J, Laux M and Wagner F 2004 Contr. Plasma Phys. 44650 - 655

[4] Otte M and Lingertat J $200229^{\text {th }}$ EPS Conference on Plasma Physics and Controlled Fusion (Montreux)

[5] Werner A Private communication

[6] Otte M, Lingertat J and Wagner F $200330^{\text {th }}$ EPS Conference on Controlled Fusion and Plasma Physics (St. Petersburg)

[7] Stangeby P C 2000 The Plasma Boundary of Magnetic Fusion Devices (Institute of Physics Publishing, Bristol and Philadelphia) 